CUBO A Mathematical Journal

Vol.16, $N^{\underline{O}} 03,(97-117)$. October 2014

\title{
Computing the inverse Laplace transform for rational functions vanishing at infinity
}

\author{
TAKAHIRO SUdo \\ Department of Mathematical Sciences, \\ Faculty of Science, University of the Ryukyus, \\ Senbaru 1, Nishihara, Okinawa 903-0213, Japan. \\ sudo@math.u-ryukyu.ac.jp
}

\begin{abstract}
We compute explicitly the inverse Laplace transform for rational functions vanishing at infinity in the general case. We also compute explicitly convolution product for continuous elementary functions involved in the general case. We then consider algebraic structure about the Laplace transform via convolution product.
\end{abstract}

\section{RESUMEN}

Calculamos explícitamente la transformada de Laplace inversa para funciones racionales que se anulan en infinito en el caso general. Además calculamos explícitamente el producto de convolución para funciones elementales continuas que participan en el caso general. Luego, consideramos estructuras algebraicas de la transformada de Laplace por medio del producto de convolución.

Keywords and Phrases: Laplace transform, rational function, convolution.

2010 AMS Mathematics Subject Classification: 44A10, 44A35, 26C15, 26A06, 26A09. 


\section{Introduction}

The Laplace transform $L(f)$ of a real-valued, function $f$ on the interval $[0, \infty)$ is defined by

$$
L(f)(s)=\int_{0}^{\infty} e^{-s t} f(t) d t
$$

for $s \in \mathbb{C}$ in the domain of convergence (cf. [2] or [3]).

The Laplace transform $L$ for continuous functions $f$ on $[0, \infty)$ is injective. This fact is known as Lerch's theorem as a fundamental theorem in the Laplace transform theory (or deduced from switching the Laplace transform as to be the Fourier transform), so that the inverse Laplace transform is well defined as the inverse image of $L(f)$ :

$$
L^{-1}(L(f))(t)=f(t)
$$

In this paper we consider real-valued, elementary functions $f$ that are defined on the real line $\mathbb{R}$ and are continuous on $\mathbb{R}$, as well as the injectivity of the Laplace transform for these continuous functions is preserved. The reason for this assumption of $\mathbb{R}$ just comes from that we do make most of the statements simplified and also that this additional symmetry induces several symmetric results and it makes it to be possible to consider the usual algebraic structure about continuous elementary functions, and is perhaps more natural than cutting down functions to be zero on the negative part of $\mathbb{R}$. But this assumption is not the same as the usual convention that $f$ is assumed to be zero on the interval $(-\infty, 0)$. Indeed, the inverse Laplace trasform defined as

$$
f(t)=\frac{1}{2 \pi i} \int_{\alpha-i \infty}^{\alpha+i \infty} e^{s t} L(f)(s) d s
$$

known as Bromwich integral requires that convention, where the complex line integral is taken for some real $\alpha$ and is computed by residue theorem as sums of residue functions. This says that even real-valued functions of one variable as well as the complex case are determined by singularities of their images under the Laplace transform. However, we do not use the complex integral in what follows.

In this paper, by elementary calculation we compute explicitly the inverse Laplace transform for rational functions vanishing at infinity in the general case and determine the inverse image. We also compute explicitly convolution product for continuous elementary functions involved in the general case. We then consider algebraic structure about the Laplace transform from our view point, which may not be written in the literature.

As a reference, there is another inductive computation known as a recursive formula for multiples of convolution in general (see [1]), but without using it we compute more explictly multiples of convolution for certain concrete continuous elementary functions.

There are 4 sections after this introduction as follows: 2. Inverse Laplace transform for rational functions in a special case; 3 . Inverse Laplace transform for rational functions in another special case; 4. Inverse Laplace transform for rational functions; 5. Algebraic structure. 
Our elemetary but explicit computation results obtained in the several general cases of those sections and our consideration and determination on the algebraic structure about the Laplace transform via convolution product would be new as well as useful and helpful as a reference.

Notation. We denote by $e^{x}$ the exponential function to the base $e$ for $x \in \mathbb{R}$ and by $\sin x$ and $\cos x$ the trigonometric functions for $x \in \mathbb{R}$. We denote by $\left(\begin{array}{l}n \\ k\end{array}\right)$ the combination of $k$ items from $n$ items mutually different.

\section{Inverse Laplace transform for rational functions in a spe- cial case}

As a well known fact, we have

Lemma 2.1. Let $\mathrm{s} \in \mathbb{C}$ with the real part $\operatorname{Re}(\mathrm{s})>0$ and $\lambda \in \mathbb{R}$ a constant with $\lambda \neq 0$. Then

$$
\mathrm{L}^{-1}\left(\frac{1}{\left(\mathrm{~s}^{2}+\lambda^{2}\right)^{2}}\right)=\frac{1}{2 \lambda^{3}} \sin \lambda t-\frac{\mathrm{t}}{2 \lambda^{2}} \cos \lambda \mathrm{t} \quad(\mathrm{t} \in \mathbb{R}) .
$$

Proof. By using a fact that the Laplace transform of the convolution product of two functions $f(t)$ and $g(t)$ is the pointwise multiplication of their Laplace transforms:

$$
L(f * g)(s)=L(f)(s) \cdot L(g)(s) \quad \text { with } \quad f * g(t)=\int_{0}^{t} f(t-\tau) g(\tau) d \tau,
$$

we compute

$$
\begin{aligned}
& \mathrm{L}^{-1}\left(\frac{1}{\left(\mathrm{~s}^{2}+\lambda^{2}\right)^{2}}\right)=\mathrm{L}^{-1}\left(\frac{1}{\mathrm{~s}^{2}+\lambda^{2}} \cdot \frac{1}{\mathrm{~s}^{2}+\lambda^{2}}\right)=\frac{1}{\lambda^{2}} \sin \lambda \mathrm{t} * \sin \lambda \mathrm{t} \\
& =\frac{1}{\lambda^{2}} \int_{0}^{\mathrm{t}} \sin \lambda(\mathrm{t}-\tau) \sin \lambda \tau \mathrm{d} \tau=\frac{1}{2 \lambda^{2}} \int_{0}^{\mathrm{t}}\{\cos \lambda(\mathrm{t}-2 \tau)-\cos \lambda \mathrm{t}\} \mathrm{d} \tau \\
& =\frac{1}{2 \lambda^{2}}\left[\frac{1}{-2 \lambda} \sin \lambda(\mathrm{t}-2 \tau)-\tau \cos \lambda \mathrm{t}\right]_{\tau=0}^{\mathrm{t}}=\frac{1}{2 \lambda^{3}} \sin \lambda \mathrm{t}-\frac{\mathrm{t}}{2 \lambda^{2}} \cos \lambda \mathrm{t} .
\end{aligned}
$$

Note that $L(\sin \lambda t)=\frac{\lambda}{s^{2}+\lambda^{2}}$ well known.

As the second step in induction, we have

Lemma 2.2. Let $s \in \mathbb{C}$ with $\operatorname{Re}(s)>0$ and $\lambda \in \mathbb{R}$ a constant with $\lambda \neq 0$. Then

$$
\mathrm{L}^{-1}\left(\frac{1}{\left(\mathrm{~s}^{2}+\lambda^{2}\right)^{3}}\right)=\left(\frac{3-\lambda^{2} \mathrm{t}^{2}}{8 \lambda^{5}}\right) \sin \lambda \mathrm{t}-\frac{3 \mathrm{t}}{8 \lambda^{4}} \cos \lambda \mathrm{t}
$$


Proof. We compute

$$
\begin{aligned}
\mathrm{L}^{-1}\left(\frac{1}{\left(\mathrm{~s}^{2}+\lambda^{2}\right)^{3}}\right) & =\mathrm{L}^{-1}\left(\frac{1}{\mathrm{~s}^{2}+\lambda^{2}} \cdot \frac{1}{\left(\mathrm{~s}^{2}+\lambda^{2}\right)^{2}}\right) \\
& =\frac{1}{\lambda} \sin \lambda \mathrm{t} * \mathrm{~L}^{-1}\left(\frac{1}{\left(\mathrm{~s}^{2}+\lambda^{2}\right)^{2}}\right)(\mathrm{t}) .
\end{aligned}
$$

Inserting the result of Lemma 2.1 above for $\mathrm{L}^{-1}\left(\frac{1}{\left(\mathrm{~s}^{2}+\lambda^{2}\right)^{2}}\right)$ and computing the convolution product by using integration by parts and addition theorem of trigonometric functions we obtain the formula in the statement.

As the third step in induction, we have

Lemma 2.3. Let $\mathrm{s} \in \mathbb{C}$ with $\operatorname{Re}(\mathrm{s})>0$ and $\lambda \in \mathbb{R}$ a constant with $\lambda \neq 0$. Then

$$
\mathrm{L}^{-1}\left(\frac{1}{\left(\mathrm{~s}^{2}+\lambda^{2}\right)^{4}}\right)=\left(\frac{5-2 \lambda^{2} \mathrm{t}^{2}}{16 \lambda^{7}}\right) \sin \lambda \mathrm{t}+\left(\frac{3^{-1} \lambda^{2} \mathrm{t}^{3}-5 \mathrm{t}}{16 \lambda^{6}}\right) \cos \lambda \mathrm{t} .
$$

Proof. We compute

$$
\begin{aligned}
\mathrm{L}^{-1}\left(\frac{1}{\left(\mathrm{~s}^{2}+\lambda^{2}\right)^{4}}\right) & =\mathrm{L}^{-1}\left(\frac{1}{\mathrm{~s}^{2}+\lambda^{2}} \cdot \frac{1}{\left(\mathrm{~s}^{2}+\lambda^{2}\right)^{3}}\right) \\
& =\frac{1}{\lambda} \sin \lambda \mathrm{t} * \mathrm{~L}^{-1}\left(\frac{1}{\left(\mathrm{~s}^{2}+\lambda^{2}\right)^{3}}\right)(\mathrm{t}) .
\end{aligned}
$$

Inserting the result of Lemma 2.2 above for $\mathrm{L}^{-1}\left(\frac{1}{\left(s^{2}+\lambda^{2}\right)^{3}}\right)$ and computing the convolution product by using integration by parts and addition theorem of trigonometric functions we obtain the formula in the statement.

Theorem 2.4. Let $\mathrm{s} \in \mathbb{C}$ with $\operatorname{Re}(\mathrm{s})>0$ and $\lambda \in \mathbb{R}$ a constant with $\lambda \neq 0$. Then, for an integer $n \geq 1$,

$$
L^{-1}\left(\frac{1}{\left(s^{2}+\lambda^{2}\right)^{2 n}}\right)=e_{2 n-2}(t) \sin \lambda t+o_{2 n-1}(t) \cos \lambda t
$$

where $\mathrm{e}_{2 \mathrm{n}-2}(\mathrm{t})$ is an even polynomial of $\mathrm{t}$ with degree $2 \mathrm{n}-2$ and with real coefficients involving $\lambda$, and $\mathrm{o}_{2 \mathrm{n}-1}(\mathrm{t})$ is an odd polynomial of $\mathrm{t}$ with degree $2 \mathrm{n}-1$ and with real coefficients involving $\lambda$. Similarly, for an integer $\mathrm{n} \geq 2$,

$$
L^{-1}\left(\frac{1}{\left(s^{2}+\lambda^{2}\right)^{2 n-1}}\right)=e_{2 n-2}(t) \sin \lambda t+o_{2 n-3}(t) \cos \lambda t .
$$

Proof. By induction, suppose that the formula for $2 \mathrm{n}$ in the statement holds. We then compute

$$
\begin{aligned}
\mathrm{L}^{-1}\left(\frac{1}{\left(\mathrm{~s}^{2}+\lambda^{2}\right)^{2 n+1}}\right) & =\mathrm{L}^{-1}\left(\frac{1}{\mathrm{~s}^{2}+\lambda^{2}} \cdot \frac{1}{\left(\mathrm{~s}^{2}+\lambda^{2}\right)^{2 n}}\right) \\
& =\frac{1}{\lambda} \sin \lambda \mathrm{t} * \mathrm{~L}^{-1}\left(\frac{1}{\left(\mathrm{~s}^{2}+\lambda^{2}\right)^{2 n}}\right)(\mathrm{t})
\end{aligned}
$$


(and inserting the formula assumed for $\mathrm{L}^{-1}\left(\frac{1}{\left(\mathrm{~s}^{2}+\lambda^{2}\right)^{2 \pi}}\right)$ we have:)

$$
\begin{aligned}
& =\frac{1}{\lambda} \sin \lambda t *\left\{e_{2 n-2}(t) \sin \lambda t+o_{2 n-1}(t) \cos \lambda t\right\} \\
& =\frac{1}{\lambda} \int_{0}^{t} \sin \lambda(t-\tau)\left\{e_{2 n-2}(\tau) \sin \lambda \tau+o_{2 n-1}(\tau) \cos \lambda \tau\right\} d \tau
\end{aligned}
$$

(and by addition theorem of trigonometric functions, we have:)

$$
\begin{aligned}
= & \frac{1}{2 \lambda} \int_{0}^{t} e_{2 n-2}(\tau)\{\cos \lambda(t-2 \tau)-\cos \lambda t\} d \tau \\
& +\frac{1}{2 \lambda} \int_{0}^{t} o_{2 n-1}(\tau)\{\sin \lambda t+\sin \lambda(t-2 \tau)\} d \tau \\
= & \frac{1}{2 \lambda}\left[\int_{0}^{t} e_{2 n-2}(\tau) \cos \lambda(t-2 \tau) d \tau-\int_{0}^{t} e_{2 n-2}(\tau) d \tau \cdot \cos \lambda t\right] \\
& +\frac{1}{2 \lambda}\left[\int_{0}^{t} o_{2 n-1}(\tau) d \tau \cdot \sin \lambda t+\int_{0}^{t} o_{2 n-1}(\tau) \sin \lambda(t-2 \tau) d \tau\right] .
\end{aligned}
$$

By using integration by parts repeatedly, we compute the first integral term among four terms as: $\int_{0}^{t} e_{2 n-2}(\tau) \cos \lambda(t-2 \tau) d \tau$

$$
\begin{aligned}
& =\left[e_{2 n-2}(\tau) \frac{\sin \lambda(t-2 \tau)}{-2 \lambda}\right]_{\tau=0}^{t}+\frac{1}{2 \lambda} \int_{0}^{t} e_{2 n-2}^{\prime}(\tau) \sin \lambda(t-2 \tau) d \tau \\
& =\left\{\frac{e_{2 n-2}(t)-e_{2 n-2}(0)}{2 \lambda}\right\} \sin \lambda t+\frac{1}{2 \lambda} \int_{0}^{t} e_{2 n-2}^{\prime}(\tau) \sin \lambda(t-2 \tau) d \tau
\end{aligned}
$$

and note that the first coefficient $\{\cdot\}$ is an even polynomial of $t$ of degree $2 n-2$, and the integral in the second term is computed as: $\int_{0}^{t} e_{2 n-2}^{\prime}(\tau) \sin \lambda(t-2 \tau) d \tau$

$$
\begin{aligned}
& =\left[e_{2 n-2}^{\prime}(\tau) \frac{-\cos \lambda(t-2 \tau)}{-2 \lambda}\right]_{\tau=0}^{t}-\frac{1}{2 \lambda} \int_{0}^{t} e_{2 n-2}^{\prime \prime}(\tau) \cos \lambda(t-2 \tau) d \tau \\
& =\left\{\frac{e_{2 n-2}^{\prime}(t)-e_{2 n-2}^{\prime}(0)}{2 \lambda}\right\} \cos \lambda t-\frac{1}{2 \lambda} \int_{0}^{t} e_{2 n-2}^{\prime \prime}(\tau) \cos \lambda(t-2 \tau) d \tau
\end{aligned}
$$

with the differential $e_{2 n-2}^{\prime}(0)=0$ and the coefficient $\{\cdot\}$ an odd polynomial of $t$ of degree $2 n-1$, and moreover, the last integral $\int_{0}^{t} e_{2 n-2}^{\prime \prime}(\tau) \cos \lambda(t-2 \tau) d \tau$ is computed inductively and finitely by integration by parts to obtain the similar coefficients of $\sin \lambda t$ and $\cos \lambda t$ summed as even and odd polynomials of $t$ with degrees less than $2 n-2$ and $2 n-1$, respectively.

The same consideration as for the first integral is applied for the fourth integral: $\int_{0}^{t} o_{2 n-1}(\tau) \sin \lambda(t-$ $2 \tau) d \tau$ to be computed as the sum of $\cos \lambda t$ and $\sin \lambda t$ with coefficients odd and even polynomials of $t$ of degree $2 n-1$ and $2 n$, respectively.

As for $\int_{0}^{t} e_{2 n-2}(\tau) d \tau \cdot \cos \lambda t$ and $\int_{0}^{t} o_{2 n-1}(\tau) d \tau \cdot \sin \lambda t$, the second and third integrals among four terms are computed to be odd and even polynomials of $t$ with degrees $2 n-1$ and $2 n-2$, respectively. 
Summing up the computations above, we obtain

$$
L^{-1}\left(\frac{1}{\left(s^{2}+\lambda^{2}\right)^{2(n+1)-1}}\right)=e_{2(n+1)-2}(t) \sin \lambda t+o_{2(n+1)-3}(t) \cos \lambda t
$$

for some even $e_{2 n}(t)$ and odd $o_{2 n-1}(t)$. This is the case where $n$ is replaced with $n+1$ in the second formula in the statement.

Similarly, the case of $2 n+2$ is deduced from the case of $2 n+1$ that now we have proved above.

Remark. Perhaps, the real coefficients involving $\lambda$ of even and odd polynomials in general $2 \mathrm{n}$ or $2 n-1$ could be determined explicitly as given in Lemmas 2.1 to 2.3 .

Corollary 2.5. For any integer $\mathrm{n} \geq 1$,

$$
\mathrm{L}^{-1}\left(\frac{1}{\left(\mathrm{~s}^{2}+\lambda^{2}\right)^{n}}\right)(\mathrm{t}) \quad \text { is an odd function as for } \mathrm{t} \in \mathbb{R} \text {. }
$$

\section{Inverse Laplace transform for rational functions in an- other special case}

As a well known fact, we have

Lemma 3.1. Let $s \in \mathbb{C}$ with $\operatorname{Re}(s)>0$ and $\lambda \in \mathbb{R}$ a constant with $\lambda \neq 0$. Then

$$
\mathrm{L}^{-1}\left(\frac{\mathrm{s}}{\left(\mathrm{s}^{2}+\lambda^{2}\right)^{2}}\right)=\frac{1}{2 \lambda} \mathrm{t} \sin \lambda \mathrm{t} \quad(\mathrm{t} \in \mathbb{R}) .
$$

Proof. We compute as in the previous section,

$$
\begin{aligned}
& L^{-1}\left(\frac{s}{\left(s^{2}+\lambda^{2}\right)^{2}}\right)=L^{-1}\left(\frac{1}{s^{2}+\lambda^{2}} \cdot \frac{s}{s^{2}+\lambda^{2}}\right)=\frac{1}{\lambda} \sin \lambda t * \cos \lambda t \\
& =\frac{1}{\lambda} \int_{0}^{t} \sin \lambda(t-\tau) \cos \lambda \tau d \tau=\frac{1}{2 \lambda} \int_{0}^{t}\{\sin \lambda(t-2 \tau)+\sin \lambda t\} d \tau \\
& =\frac{1}{2 \lambda}\left[\frac{1}{2 \lambda} \cos \lambda(t-2 \tau)+\tau \sin \lambda t\right]_{\tau=0}^{t}=\frac{1}{2 \lambda} t \sin \lambda t .
\end{aligned}
$$

Note that $L(\cos \lambda t)=\frac{s}{s^{2}+\lambda^{2}}$ well known.

As the second step in induction, we have

Lemma 3.2. Let $\mathrm{s} \in \mathbb{C}$ with $\operatorname{Re}(\mathrm{s})>0$ and $\lambda \in \mathbb{R}$ a constant with $\lambda \neq 0$. Then

$$
\mathrm{L}^{-1}\left(\frac{\mathrm{s}}{\left(\mathrm{s}^{2}+\lambda^{2}\right)^{3}}\right)=\left(\frac{1+\lambda^{2} \mathrm{t}^{2}}{4 \lambda^{3}}\right) \sin \lambda \mathrm{t}-\frac{\mathrm{t}}{2 \lambda} \cos \lambda \mathrm{t} .
$$


Proof. We compute

$$
\begin{aligned}
\mathrm{L}^{-1}\left(\frac{\mathrm{s}}{\left(\mathrm{s}^{2}+\lambda^{2}\right)^{3}}\right) & =\mathrm{L}^{-1}\left(\frac{1}{\mathrm{~s}^{2}+\lambda^{2}} \cdot \frac{\mathrm{s}}{\left(\mathrm{s}^{2}+\lambda^{2}\right)^{2}}\right) \\
& =\frac{1}{\lambda} \sin \lambda \mathrm{t} * \mathrm{~L}^{-1}\left(\frac{\mathrm{s}}{\left(\mathrm{s}^{2}+\lambda^{2}\right)^{2}}\right)(\mathrm{t}) .
\end{aligned}
$$

Inserting the result of Lemma 3.1 above for $\mathrm{L}^{-1}\left(\frac{\mathrm{s}}{\left(\mathrm{s}^{2}+\lambda^{2}\right)^{2}}\right)$ and computing the convolution product by using integration by parts and addition theorem of trigonometric functions we obtain the formula in the statement.

One can use the following decomposition and Lemma 2.1 in the previous section:

$$
\mathrm{L}^{-1}\left(\frac{\mathrm{s}}{\mathrm{s}^{2}+\lambda^{2}} \cdot \frac{1}{\left(\mathrm{~s}^{2}+\lambda^{2}\right)^{2}}\right)=\cos \lambda \mathrm{t} * \mathrm{~L}^{-1}\left(\frac{1}{\left(\mathrm{~s}^{2}+\lambda^{2}\right)^{2}}\right)(\mathrm{t})
$$

As the third step in induction, we have

Lemma 3.3. Let $s \in \mathbb{C}$ with $\operatorname{Re}(s)>0$ and $\lambda \in \mathbb{R}$ a constant with $\lambda \neq 0$. Then

$$
\mathrm{L}^{-1}\left(\frac{\mathrm{s}}{\left(\mathrm{s}^{2}+\lambda^{2}\right)^{4}}\right)=\left(\frac{2+\lambda-\lambda^{3} \mathrm{t}^{2}}{16 \lambda^{5}}\right) \sin \lambda \mathrm{t}+\left(\frac{-3 \lambda \mathrm{t}-3^{-1} 2 \lambda^{3} \mathrm{t}^{3}}{16 \lambda^{4}}\right) \cos \lambda \mathrm{t} .
$$

Proof. We compute

$$
\begin{aligned}
\mathrm{L}^{-1}\left(\frac{\mathrm{s}}{\left(\mathrm{s}^{2}+\lambda^{2}\right)^{4}}\right) & =\mathrm{L}^{-1}\left(\frac{1}{\mathrm{~s}^{2}+\lambda^{2}} \cdot \frac{\mathrm{s}}{\left(\mathrm{s}^{2}+\lambda^{2}\right)^{3}}\right) \\
& =\frac{1}{\lambda} \sin \lambda \mathrm{t} * \mathrm{~L}^{-1}\left(\frac{\mathrm{s}}{\left(\mathrm{s}^{2}+\lambda^{2}\right)^{3}}\right)(\mathrm{t}) .
\end{aligned}
$$

Inserting the result of Lemma 3.2 above for $\mathrm{L}^{-1}\left(\frac{\mathrm{s}}{\left(\mathrm{s}^{2}+\lambda^{2}\right)^{3}}\right)$ and computing the convolution product by using integration by parts and addition theorem of trigonometric functions we obtain the formula in the statement.

Theorem 3.4. Let $s \in \mathbb{C}$ with $\operatorname{Re}(s)>0$ and $\lambda \in \mathbb{R}$ a constant with $\lambda \neq 0$. Then, for an integer $n \geq 2$,

$$
L^{-1}\left(\frac{s}{\left(s^{2}+\lambda^{2}\right)^{2 n}}\right)=e_{2 n-2}(t) \sin \lambda t+o_{2 n-1}(t) \cos \lambda t
$$

where $\mathrm{e}_{2 \mathrm{n}-2}(\mathrm{t})$ is an even polynomial of $\mathrm{t}$ with degree $2 \mathrm{n}-2$ and with real coefficients involving $\lambda$, and $\mathrm{o}_{2 \mathrm{n}-1}(\mathrm{t})$ is an odd polynomial of $\mathrm{t}$ with degree $2 \mathrm{n}-1$ and with real coefficients involving $\lambda$. Similarly, for an integer $\mathrm{n} \geq 2$,

$$
L^{-1}\left(\frac{s}{\left(s^{2}+\lambda^{2}\right)^{2 n-1}}\right)=e_{2 n-2}(t) \sin \lambda t+o_{2 n-3}(t) \cos \lambda t
$$


Proof. By induction, suppose that two formula in the statement hold for $2 \mathrm{n}$. We then compute

$$
\begin{aligned}
\mathrm{L}^{-1}\left(\frac{\mathrm{s}}{\left(\mathrm{s}^{2}+\lambda^{2}\right)^{2 n+1}}\right) & =\mathrm{L}^{-1}\left(\frac{1}{\mathrm{~s}^{2}+\lambda^{2}} \cdot \frac{\mathrm{s}}{\left(\mathrm{s}^{2}+\lambda^{2}\right)^{2 n}}\right) \\
& =\frac{1}{\lambda} \sin \lambda \mathrm{t} * \mathrm{~L}^{-1}\left(\frac{\mathrm{s}}{\left(\mathrm{s}^{2}+\lambda^{2}\right)^{2 n}}\right)(\mathrm{t})
\end{aligned}
$$

and inserting the formula assumed for $\mathrm{L}^{-1}\left(\frac{s}{\left(s^{2}+\lambda^{2}\right)^{2 n}}\right)$ we have

$$
\begin{aligned}
& =\frac{1}{\lambda} \sin \lambda t *\left\{e_{2 n-2}(t) \sin \lambda t+o_{2 n-1}(t) \cos \lambda t\right\} \\
& =\frac{1}{\lambda} \int_{0}^{t} \sin \lambda(t-\tau)\left\{e_{2 n-2}(\tau) \sin \lambda \tau+o_{2 n-1}(\tau) \cos \lambda \tau\right\} d \tau .
\end{aligned}
$$

Note that this integral is exactly the same as the integral in the case of $\mathrm{L}^{-1}\left(\frac{1}{\left(s^{2}+\lambda^{2}\right)^{2 n+1}}\right)$ in the proof of Theorem 2.4. Thus, we omit the rest of the proof.

Similarly, the case of $2 n+2$ is deduced from the case of $2 n+1$ that now we have proved.

Corollary 3.5. For any integer $\mathrm{n} \geq 2$,

$$
\mathrm{L}^{-1}\left(\frac{\mathrm{s}}{\left(\mathrm{s}^{2}+\lambda^{2}\right)^{n}}\right)(\mathrm{t}) \quad \text { is an odd function as for } \mathrm{t} \in \mathbb{R} \text {. }
$$

Remark. Note that the polynomials obtained in Theorem 3.4 are not the same as those in Theorem 2.4, but we use the same symbols for both of the polynomials.

Anyhow, combining both of Theorem 2.4 and Theorem 3.4 we get

Corollary 3.6. Both $\mathrm{L}^{-1}\left(\frac{1}{\left(s^{2}+\lambda^{2}\right)^{2 n}}\right)$ for $\mathrm{n} \geq 1$ and $\mathrm{L}^{-1}\left(\frac{\mathrm{s}}{\left(\mathrm{s}^{2}+\lambda^{2}\right)^{2 n}}\right)$ for $\mathrm{n} \geq 2$ are written as the same form:

$$
e_{2 n-2}(t) \sin \lambda t+o_{2 n-1}(t) \cos \lambda t
$$

and both $\mathrm{L}^{-1}\left(\frac{1}{\left(\mathrm{~s}^{2}+\lambda^{2}\right)^{2 n-1}}\right)$ for $\mathrm{n} \geq 1$ and $\mathrm{L}^{-1}\left(\frac{\mathrm{s}}{\left(\mathrm{s}^{2}+\lambda^{2}\right)^{2 n-1}}\right)$ for $\mathrm{n} \geq 2$ are written as the same form:

$$
e_{2 n-2}(t) \sin \lambda t+o_{2 n-3}(t) \cos \lambda t
$$

\section{Inverse Laplace transform for rational functions}

It is well known that a rational function $f$ of $s \in \mathbb{C}$ such that $f(s)=\frac{p(s)}{q(s)}$ with $p(s), q(s)$ polynomials of $s \in \mathbb{C}$ with real coefficients and with $\operatorname{deg} p(s)<\operatorname{deg} q(s)$ is decomposed into partial fractions as:

$$
\begin{aligned}
& f(s)=\sum_{j=1}^{m_{0}} \frac{c_{0 j}}{s^{j}}+\sum_{k=1}^{l_{1}} \sum_{j=1}^{m_{k}} \frac{c_{k j}}{\left(s-a_{k}\right)^{j}} \\
& +\sum_{k=1}^{l_{2}} \sum_{j=1}^{n_{k}} \frac{d_{k j} s}{\left(\left(s-b_{k}\right)^{2}+c_{k}^{2}\right)^{j}}+\sum_{k=1}^{l_{2}} \sum_{j=1}^{n_{k}} \frac{e_{k j}}{\left(\left(s-b_{k}\right)^{2}+c_{k}^{2}\right)^{j}}, \\
& \text { where } \quad q(s)=q_{0} s^{m_{0}} \prod_{k=1}^{l_{1}}\left(s-a_{k}\right)^{m_{k}} \prod_{k=1}^{l_{2}}\left(\left(s-b_{k}\right)^{2}+c_{k}^{2}\right)^{n_{k}}
\end{aligned}
$$


the factorization of $q(s)$ in real $\mathbb{R}$ with $a_{k}$ real roots of multiplicity $m_{k}$ and with $b_{k} \pm i c_{k}$ imaginary roots of multiplicity $n_{k}$, for some $q_{0} \neq 0$ in $\mathbb{R}, m_{0} \geq 0, m_{k} \geq 1, n_{k} \geq 1$, and $a_{k} \neq 0, b_{k} \neq 0$ or $0, c_{k} \neq 0$ in $\mathbb{R}$, and $l_{1}, l_{2} \in \mathbb{N}$, and $c_{0 j}, c_{k j} \in \mathbb{R}$ but $c_{0 m_{0}} \neq 0$ or $c_{k m_{k}} \neq 0$ corresponding to the highest terms if nonzero; and $d_{k j}, e_{k j} \in \mathbb{R}$ but either $d_{k n_{k}} \neq 0$ or $e_{k n_{k}} \neq 0$ in $\mathbb{R}$ corresponding to the highest terms if nonzero.

Therefore, using the basic facts of Laplace transform for polynomials and translation and our results in the previous sections we obtain

Theorem 4.1. Let $\mathrm{f}(\mathrm{s})$ be a rational function of $\mathrm{s} \in \mathbb{C}$ vanishing at infinity with the factorization as above. Assume that the real part of s satisfies the following inequality:

$$
\operatorname{Re}(s)>\max \left\{0, a_{k}, b_{j} \mid 1 \leq k \leq l_{1}, 1 \leq j \leq l_{2}\right\}
$$

if $m_{0} \geq 1, l_{1} \geq 1$, and $l_{2} \geq 1$, and otherwise, some elements of the set may be dropped. Then, in general,

$$
\begin{aligned}
& L^{-1}(f(s))(t)=\sum_{j=1}^{m_{0}} \frac{c_{0 j} t^{j-1}}{(j-1) !}+\sum_{k=1}^{l_{1}} \sum_{j=1}^{m_{k}} \frac{c_{k j} e^{a_{k} t_{t} t^{j-1}}}{(j-1) !} \\
& +\sum_{k=1}^{l_{2}} \frac{d_{k 1}}{c_{k}} e^{b_{k} t} \sin c_{k} t+\sum_{k=1}^{l_{2}} \frac{d_{k 2}}{2 c_{k}} e^{b_{k} t} t \sin c_{k} t \\
& +\sum_{k=1}^{l_{2}} \sum_{n=2}^{\left\lceil\frac{n_{k}}{2}\right\rceil} d_{k(2 n-1)} e^{b_{k} t}\left[e_{k(2 n-2)}(t) \sin c_{k} t+o_{k(2 n-3)}(t) \cos c_{k} t\right] \\
& +\sum_{k=1}^{l_{2}} \sum_{n=2}^{\left\lfloor\frac{n_{k}}{2}\right\rfloor} d_{k(2 n)} e^{b_{k} t}\left[e_{k(2 n-2)}(t) \sin c_{k} t+o_{k(2 n-1)}(t) \cos c_{k} t\right] \\
& +\sum_{k=1}^{\mathrm{l}_{2}} e_{\mathrm{k} 1} e^{\mathrm{b}_{\mathrm{k}} \mathrm{t}} \cos \mathrm{c}_{\mathrm{k}} \mathrm{t} \\
& +\sum_{k=1}^{\mathrm{l}_{2}} \sum_{n=2}^{\left\lceil\frac{n_{k}}{2}\right\rceil} e_{k(2 n-1)} e^{b_{k} t}\left[e_{k(2 n-2)}^{\sim}(t) \sin c_{k} t+o_{k(2 n-3)}^{\sim}(t) \cos c_{k} t\right]
\end{aligned}
$$

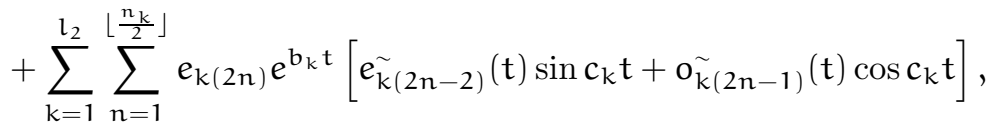

where $\lceil\mathrm{x}\rceil$ means the minimum integer $\mathrm{y}$ such that $\mathrm{y} \geq \mathrm{x}$, and $\lfloor\mathrm{x}\rfloor$ means the maximum integer $\mathrm{y}$ such that $\mathrm{y} \leq \mathrm{x}$, and $\mathrm{e}_{\mathrm{kj}}(\mathrm{t}), \mathrm{o}_{\mathrm{kj}}(\mathrm{t}), \mathrm{e}_{\mathrm{kj}}^{\sim}(\mathrm{t})$, and $\tilde{\mathrm{o}}_{\mathrm{kj}}(\mathrm{t})$ are polynomials of $\mathrm{t}$ with degree $\mathrm{j}$ and with real coefficients involving $\mathrm{c}_{\mathrm{k}}$.

Remark. The restriction on $s \in \mathbb{C}$ comes from the existence of the Laplace transform $\mathrm{L}(\mathrm{g}(\mathrm{t}))(\mathrm{s})=$ $f(s)$ for some $g(t)$. Namely, $s \in \mathbb{C}$ should belong to a domain of convergence of $L(g(t))(s)$. Note that the maximum in the statement is just that of real parts of poles of the rational function $f(s)$ given. 
Remark. Note that $\mathrm{L}(\delta(\mathrm{t}))=1$ for $\delta(\mathrm{t})$ the Dirac function, viewed as a distribution, i.e. a functional, so that $\mathrm{L}^{-1}(1)=\delta(\mathrm{t})$, where the constant unit function 1 on $\mathbb{C}$ is also viewed as a distribution.

\section{$5 \quad$ Algebraic structure}

In this section we consider algebraic structure about the Laplace transform via convolution product.

We denote by $R_{0}(\mathbb{C})$ the set of rational functions on $\mathbb{C}$ with real coefficients, vanishing at infinity. Under pointwise addition and multiplication, $R_{0}(\mathbb{C})$ becomes a non-unital algebra over $\mathbb{R}$. Indeed,

Lemma 5.1. The set $R_{0}(\mathbb{C})$ is an algebra over $\mathbb{R}$ under point-wise operations.

Proof. Let $f, g \in R_{0}(\mathbb{C})$ such that $f(s)=\frac{p_{1}(s)}{q_{1}(s)}$ and $g(s)=\frac{p_{2}(s)}{q_{2}(s)}$ for some polynomials $p_{j}(s), q_{j}(s)$ with $\operatorname{deg} p_{j}<\operatorname{deg} q_{j}(j=1,2)$. Then

$$
\begin{aligned}
& \qquad f(s)+g(s)=\frac{p_{1}(s) q_{2}(s)+p_{2}(s) q_{1}(s)}{q_{1}(s) q_{2}(s)} \in R_{0}(\mathbb{C}) \\
& \text { and } f(s) g(s)=\frac{p_{1}(s) p_{2}(s)}{q_{1}(s) q_{2}(s)} \in R_{0}(\mathbb{C})
\end{aligned}
$$

and other axioms can be also easily checked.

We denote by $A(\mathbb{R})$ the algebra over $\mathbb{R}$ generated by the sets of elementary continuous functions on $\mathbb{R}:\left\{\mathrm{t}^{\mathrm{n}} \mid \mathrm{n} \in \mathbb{N}=\{0,1,2, \cdots\}\right\}$ of monomials and $\left\{\mathrm{e}^{\mu \mathrm{t}} \mid \mu \in \mathbb{R}\right\}$ and $\{\sin \lambda \mathrm{t}, \cos \lambda \mathrm{t} \mid \lambda \in \mathbb{R}\}$ under point-wise addition and point-wise multiplication. Under (extended) convolution product defined as:

$$
(f * g)(t)=\int_{0}^{t} f(t-\tau) g(\tau) d \tau, \quad(t \in \mathbb{R})
$$

for $f, g \in A(\mathbb{R})$, which is a commutative and associative operation as well known as the usual case on $[0, \infty), A(\mathbb{R})$ becomes an algebra over $\mathbb{R}$. Indeed, check it in details as follows:

Proposition 5.2. The real algebra $A(\mathbb{R})$ under point-wise operations is viewed as an algebra over $\mathbb{R}$ under convolution product, as given in the following:

$$
\text { (1) }\left(t^{n} * t^{m}\right)(t)=\left[\sum_{k=0}^{n}\left(\begin{array}{l}
n \\
k
\end{array}\right) \frac{(-1)^{k}}{k+m+1}\right] t^{n+m+1}
$$

for $n, m \in \mathbb{N} ;$ and for $\mu, \lambda \in \mathbb{R}$ with $\mu \neq \lambda$,

$$
\text { (2) } \quad\left(e^{\mu t} * e^{\lambda t}\right)(t)= \begin{cases}\frac{e^{\lambda t}-e^{\mu t}}{\lambda-\mu} & \text { if } \mu \neq \lambda, \\ t e^{\mu t} & \text { if } \mu=\lambda ;\end{cases}
$$


and for $\mathrm{n} \geq 0$ in $\mathbb{N}$ and $\mu \in \mathbb{R}$ with $\mu \neq 0$,

$$
\text { (3) } \quad\left(t^{n} * e^{\mu t}\right)(t)=\sum_{k=0}^{n}\left(\begin{array}{l}
n \\
k
\end{array}\right) t^{n-k}(-k) \int_{0}^{t} \tau^{k} e^{\mu \tau} d \tau
$$

with the integral $\int_{0}^{\mathrm{t}} \tau^{\mathrm{k}} e^{\mu \tau} \mathrm{d} \tau$ equal to

$$
e^{\mu t} \sum_{l=1}^{k+1} \frac{(-1)^{l-1} k !}{\mu^{l}(k+1-l) !} t^{k+1-l}+\left(\frac{(-1)^{k+1} k !}{\mu^{k+1}}\right)
$$

and for $\lambda, \mu \in \mathbb{R}$,

$$
\text { (4) }(\sin \lambda t * \cos \mu t)(t)= \begin{cases}\frac{\lambda}{\lambda^{2}-\mu^{2}} \cos \mu t-\frac{\lambda}{\lambda^{2}-\mu^{2}} \cos \lambda t & \text { if } \lambda \neq \pm \mu, \\ \frac{1}{2} t \sin \lambda t & \text { if } \lambda= \pm \mu,\end{cases}
$$

and

and

$$
(\cos \lambda t * \cos \mu t)(t)= \begin{cases}\frac{\mu}{\lambda^{2}-\mu^{2}} \sin \mu t-\frac{\lambda}{\lambda^{2}-\mu^{2}} \sin \lambda t & \text { if } \lambda \neq \pm \mu, \\ \frac{1}{2} t \cos \lambda t+\frac{1}{2 \lambda} \sin \lambda t & \text { if } \lambda= \pm \mu,\end{cases}
$$

$$
(\sin \lambda t * \sin \mu t)(t)= \begin{cases}\frac{\lambda}{\lambda^{2}-\mu^{2}} \sin \mu t-\frac{\mu}{\lambda^{2}-\mu^{2}} \sin \lambda t & \text { if } \lambda \neq \pm \mu, \\ \frac{-1}{2} t \cos \lambda t+\frac{1}{2 \lambda} \sin \lambda t & \text { if } \lambda= \pm \mu ;\end{cases}
$$

and moreover, for $\mu, \lambda \in \mathbb{R}$ with $\mu \neq 0$,

$$
\text { (5) } \quad\left(e^{\mu t} * \sin \lambda t\right)(t)=\frac{1}{\lambda^{2}+\mu^{2}}\left\{\lambda e^{-\mu t}-\mu \sin \lambda t-\lambda \cos \lambda t\right\} .
$$

and

$$
\left(e^{\mu t} * \cos \lambda t\right)(t)=\frac{1}{\lambda^{2}+\mu^{2}}\left\{\mu e^{-\mu t}-\mu \cos \lambda t+\lambda \sin \lambda t\right\}
$$

and furthermore,

$$
\text { (6) } \quad\left(t^{n} * \sin \lambda t\right)(t)=\sum_{k=0}^{n}\left(\begin{array}{l}
n \\
k
\end{array}\right) t^{n-k}(-1)^{k} \int_{0}^{t} \tau^{k} \sin \lambda \tau d \tau
$$

with $\mathrm{I}_{\mathrm{k}}=\int_{0}^{\mathrm{t}} \tau^{\mathrm{k}} \sin \lambda \tau \mathrm{d} \tau$ given by, for $\mathrm{m} \in \mathbb{N}$ with $\mathrm{m} \geq 0$, if $\mathrm{k}=2 \mathrm{~m}$,

$$
\begin{aligned}
I_{2 m}= & \sum_{l=1}^{m+1} \frac{(-1)^{l} k !}{\lambda^{2 l-1}(k-2 l+2) !} t^{k-2 l+2} \cos \lambda t \\
& +\sum_{l=1}^{m} \frac{(-1)^{(l-1)} k !}{\lambda^{2 l}(k-2 l+1) !} t^{k-2 l+1} \sin \lambda t+\frac{(-1)^{m} k !}{\lambda^{2 m+1}}
\end{aligned}
$$

and if $\mathrm{k}=2 \mathrm{~m}+1$,

$$
\begin{aligned}
\mathrm{I}_{2 \mathrm{~m}+1}= & \sum_{l=1}^{\mathrm{m}+1} \frac{(-1)^{\mathrm{l} k} \mathrm{k}}{\lambda^{2 l-1}(k-2 l+2) !} \mathrm{t}^{k-2 l+2} \cos \lambda \mathrm{t} \\
& +\sum_{l=1}^{m+1} \frac{(-1)^{(l-1)} k !}{\lambda^{2 l}(k-2 l+1) !} \mathrm{t}^{k-2 l+1} \sin \lambda t
\end{aligned}
$$


so that

$$
\begin{aligned}
& \left(t^{n} * \sin \lambda t\right)(t)=\sum_{m=0}^{\left\lfloor\frac{n}{2}\right\rfloor}\left(\begin{array}{c}
n \\
2 m
\end{array}\right)\left\{\sum_{l=1}^{m+1} \frac{(-1)^{2 m+l} k !}{\lambda^{2 l-1}(k-2 l+2) !} t^{n-2 l+2} \cos \lambda t\right. \\
& \left.+\sum_{l=1}^{m} \frac{(-1)^{(2 m+l-1)} k !}{\lambda^{2 l}(k-2 l+1) !} t^{n-2 l+1} \sin \lambda t+\frac{(-1)^{3 m} k !}{\lambda^{2 m+1}} t^{n-2 m}\right\} \\
& +\sum_{m=0}^{\left\lceil\frac{n}{2}\right\rceil-1}\left(\begin{array}{c}
n \\
2 m+1
\end{array}\right)\left\{\sum_{l=1}^{m+1} \frac{(-1)^{2 m+1+l} k !}{\lambda^{2 l-1}(k-2 l+2) !} t^{n-2 l+2} \cos \lambda t\right. \\
& \left.+\sum_{l=1}^{m+1} \frac{(-1)^{(2 m+l)} k !}{\lambda^{2 l}(k-2 l+1) !} t^{n-2 l+1} \sin \lambda t\right\}
\end{aligned}
$$

and also,

$$
\left(t^{n} * \cos \lambda t\right)(t)=\sum_{k=0}^{n}\left(\begin{array}{l}
n \\
k
\end{array}\right) t^{n-k}(-1)^{k} \int_{0}^{t} \tau^{k} \cos \lambda \tau d \tau
$$

with $\mathrm{J}_{\mathrm{k}} \equiv \int_{0}^{\mathrm{t}} \tau^{\mathrm{k}} \cos \lambda \tau \mathrm{d} \tau$ given by, for $\mathrm{m} \in \mathbb{N}$ with $\mathrm{m} \geq 0$, if $\mathrm{k}=2 \mathrm{~m}$,

$$
\begin{aligned}
J_{2 m}= & \sum_{l=1}^{m+1} \frac{(-1)^{(l-1)} k !}{\lambda^{2 l-1}(k-2 l+2) !} \mathrm{t}^{k-2 l+2} \sin \lambda t \\
& +\sum_{l=1}^{m} \frac{(-1)^{(l-1)} k !}{\lambda^{2 l}(k-2 l+1) !} \mathrm{t}^{k-2 l+1} \cos \lambda t
\end{aligned}
$$

and if $\mathrm{k}=2 \mathrm{~m}+1$,

$$
\begin{aligned}
J_{2 m+1}= & \sum_{l=1}^{m+1} \frac{(-1)^{(l-1)} k !}{\lambda^{2 l-1}(k-2 l+2) !} t^{k-2 l+2} \sin \lambda t \\
& +\sum_{l=1}^{m+1} \frac{(-1)^{(l-1)} k !}{\lambda^{2 l}(k-2 l+1) !} t^{k-2 l+1} \cos \lambda t+\frac{(-1)^{m+1} k !}{\lambda^{2 m+2}}
\end{aligned}
$$

so that

$$
\begin{aligned}
& \left(t^{n} * \cos \lambda t\right)(t)=\sum_{m=0}^{\left\lfloor\frac{n}{2}\right\rfloor}\left(\begin{array}{c}
n \\
2 m
\end{array}\right)\left\{\sum_{l=1}^{m+1} \frac{(-1)^{(2 m+l)} k !}{\lambda^{2 l-1}(k-2 l+2) !} t^{n-2 l+2} \sin \lambda t\right. \\
& \left.+\sum_{l=1}^{m} \frac{(-1)^{(2 m+l)} k !}{\lambda^{2 l}(k-2 l+1) !} t^{n-2 l+1} \cos \lambda t\right\} \\
& +\sum_{m=0}^{\left\lceil\frac{n}{2}\right\rceil-1}\left(\begin{array}{c}
n \\
2 m+1
\end{array}\right)\left\{\sum_{l=1}^{m+1} \frac{(-1)^{(2 m+l)} k !}{\lambda^{2 l-1}(k-2 l+2) !} t^{n-2 l+2} \sin \lambda t\right. \\
& \left.+\sum_{l=1}^{m+1} \frac{(-1)^{(2 m+l)} k !}{\lambda^{2 l}(k-2 l+1) !} t^{n-2 l+1} \cos \lambda t+\frac{(-1)^{3 m+2} k !}{\lambda^{2 m+2}} t^{n-2 m-1}\right\} .
\end{aligned}
$$


Finally, the convolution of general monomials is given by, as an example,

$$
\begin{aligned}
& \text { (7) }\left(t^{n} e^{\mu_{1} t} \sin \lambda_{1} t\right) *\left(t^{m} e^{\mu_{2} t} \cos \lambda_{2} t\right)(t) \\
& =e^{\mu_{1} t} \sum_{k=0}^{n}\left(\begin{array}{l}
n \\
k
\end{array}\right) t^{n-k}(-1)^{k} \int_{0}^{t} \tau^{k+m} e^{\left(\mu_{2}-\mu_{1}\right) \tau} \sin \lambda_{1}(t-\tau) \cos \lambda_{2} \tau d \tau
\end{aligned}
$$

with $\sin \lambda_{1}(t-\tau) \cos \lambda_{2} \tau=\frac{1}{2}\left\{\sin \left(\lambda_{1} t-\left(\lambda_{1}-\lambda_{2}\right) \tau\right)+\sin \left(\lambda_{1} t-\left(\lambda_{1}+\lambda_{2}\right) \tau\right)\right\}$, and then the following integral is computed inductively as

$$
\begin{aligned}
& \mathrm{I}_{\mathrm{k}+\mathrm{m}, \sin } \equiv \int_{0}^{\mathrm{t}} \tau^{\mathrm{k}+\mathrm{m}} e^{\left(\mu_{2}-\mu_{1}\right) \tau} \sin \left(\lambda_{1} \mathrm{t}-\left(\lambda_{1} \pm \lambda_{2}\right) \tau\right) \mathrm{d} \tau \\
&=\frac{\mu_{2}-\mu_{1}}{\left(\mu_{2}-\mu_{1}\right)^{2}+\left(\lambda_{1} \pm \lambda_{2}\right)^{2}} \mathrm{t}^{\mathrm{k}+\mathrm{m}} e^{\left(\mu_{2}-\mu_{1}\right) \mathrm{t}} \sin \left(\mp \lambda_{2} \mathrm{t}\right) \\
& \quad+\frac{\lambda_{1} \pm \lambda_{2}}{\left(\mu_{2}-\mu_{1}\right)^{2}+\left(\lambda_{1} \pm \lambda_{2}\right)^{2}} \mathrm{t}^{\mathrm{k}+\mathrm{m}} e^{\left(\mu_{2}-\mu_{1}\right) \mathrm{t}} \cos \left(\mp \lambda_{2} \mathrm{t}\right) \\
& \quad-\frac{(k+m)\left(\mu_{2}-\mu_{1}\right)}{\left(\mu_{2}-\mu_{1}\right)^{2}+\left(\lambda_{1} \pm \lambda_{2}\right)^{2}} \int_{0}^{t} \tau^{k+m-1} e^{\left(\mu_{2}-\mu_{1}\right) \tau} \sin \left(\lambda_{1} t-\left(\lambda_{1} \pm \lambda_{2}\right) \tau\right) d \tau \\
&-\frac{(k+m)\left(\lambda_{1} \pm \lambda_{2}\right)}{\left(\mu_{2}-\mu_{1}\right)^{2}+\left(\lambda_{1} \pm \lambda_{2}\right)^{2}} \int_{0}^{t} \tau^{k+m-1} e^{\left(\mu_{2}-\mu_{1}\right) \tau} \cos \left(\lambda_{1} t-\left(\lambda_{1} \pm \lambda_{2}\right) \tau\right) d \tau,
\end{aligned}
$$

where the last two integrals are denoted by $\mathrm{I}_{\mathrm{k}+\mathrm{m}-1, \sin }$ and $\mathrm{I}_{\mathrm{k}+\mathrm{m}-1, \cos }$ respectively, and the integrals can be inductively reduced to the cases of $\mathrm{I}_{j, \sin }$ and $\mathrm{I}_{\mathbf{j}, \cos }$ for $1 \leq \mathrm{j} \leq \mathrm{k}+\mathrm{m}-2$ and finally to the case of $\mathrm{I}_{0, \sin }$ and $\mathrm{I}_{0, \cos }$ that are given by

$$
\begin{aligned}
& \mathrm{I}_{0, \sin } \equiv \int_{0}^{\mathrm{t}} e^{\left(\mu_{2}-\mu_{1}\right) \tau} \sin \left(\lambda_{1} t-\left(\lambda_{1} \pm \lambda_{2}\right) \tau\right) d \tau \\
& =\frac{\mu_{2}-\mu_{1}}{\left(\mu_{2}-\mu_{1}\right)^{2}+\left(\lambda_{1} \pm \lambda_{2}\right)^{2}}\left\{e^{\left(\mu_{2}-\mu_{1}\right) t} \sin \left(\mp \lambda_{2} t\right)-\sin \left(\lambda_{1} t\right)\right\} \\
& \quad+\frac{\lambda_{1} \pm \lambda_{2}}{\left(\mu_{2}-\mu_{1}\right)^{2}+\left(\lambda_{1} \pm \lambda_{2}\right)^{2}}\left\{e^{\left(\mu_{2}-\mu_{1}\right) t} \cos \left(\mp \lambda_{2} t\right)-\cos \left(\lambda_{1} t\right)\right\}
\end{aligned}
$$

and

$$
\begin{aligned}
& \mathrm{I}_{0, \cos } \equiv \int_{0}^{t} e^{\left(\mu_{2}-\mu_{1}\right) \tau} \cos \left(\lambda_{1} t-\left(\lambda_{1} \pm \lambda_{2}\right) \tau\right) d \tau \\
& =\frac{\mu_{2}-\mu_{1}}{\left(\mu_{2}-\mu_{1}\right)^{2}+\left(\lambda_{1} \pm \lambda_{2}\right)^{2}}\left\{e^{\left(\mu_{2}-\mu_{1}\right) t} \cos \left(\mp \lambda_{2} t\right)-\cos \left(\lambda_{1} t\right)\right\} \\
& \quad-\frac{\lambda_{1} \pm \lambda_{2}}{\left(\mu_{2}-\mu_{1}\right)^{2}+\left(\lambda_{1} \pm \lambda_{2}\right)^{2}}\left\{e^{\left(\mu_{2}-\mu_{1}\right) t} \sin \left(\mp \lambda_{2} t\right)-\sin \left(\lambda_{1} t\right)\right\}
\end{aligned}
$$

Other cases of convolution products of general monomials with $\sin$ and $\cos$ changed are also computed similarly, but omitted. 
Proof. For (1), check first that for $n, m \geq 0$ in $\mathbb{N}$,

$$
\begin{aligned}
& \left(t^{n} * t^{m}\right)(t)=\int_{0}^{t}(t-\tau)^{n} \tau^{m} d \tau \\
& =\int_{0}^{t} \sum_{k=0}^{n}\left(\begin{array}{l}
n \\
k
\end{array}\right) t^{n-k}(-1)^{k} \tau^{k+m} d \tau=\sum_{k=0}^{n}\left(\begin{array}{l}
n \\
k
\end{array}\right) t^{n-k}(-1)^{k} \frac{t^{k+m+1}}{k+m+1} \\
& =\left[\sum_{k=0}^{n}\left(\begin{array}{l}
n \\
k
\end{array}\right) \frac{(-1)^{k}}{k+m+1}\right] t^{n+m+1} \in A(\mathbb{R}) .
\end{aligned}
$$

Also, for (2), for $\mu, \lambda \in \mathbb{R}$ with $\mu \neq \lambda$,

$$
\begin{aligned}
\left(e^{\mu t} * e^{\lambda t}\right)(t) & =\int_{0}^{t} e^{\mu(t-\tau)} e^{\lambda \tau} d \tau=e^{\mu t} \int_{0}^{t} e^{(\lambda-\mu) \tau} d \tau \\
& =e^{\mu t}\left[\frac{e^{(\lambda-\mu) \tau}}{\lambda-\mu}\right]_{\tau=0}^{t}=\frac{e^{\lambda t}-e^{\mu t}}{\lambda-\mu} \in A(\mathbb{R}) .
\end{aligned}
$$

If $\mu=\lambda$, then

$$
\left(e^{\mu t} * e^{\lambda t}\right)(t)=\int_{0}^{t} e^{\mu t} d \tau=t e^{\mu t} \in A(\mathbb{R}) .
$$

Moreover, for (3), for $n \geq 0$ in $\mathbb{N}$ and $\mu \in \mathbb{R}$ with $\mu \neq 0$,

$$
\begin{aligned}
& \left(t^{n} * e^{\mu t}\right)(t)=\int_{0}^{t}(t-\tau)^{n} e^{\mu \tau} d \tau \\
& =\int_{0}^{t} \sum_{k=0}^{n}\left(\begin{array}{l}
n \\
k
\end{array}\right) t^{n-k}(-k) \tau^{k} e^{\mu \tau} d \tau=\sum_{k=0}^{n}\left(\begin{array}{l}
n \\
k
\end{array}\right) t^{n-k}(-k) \int_{0}^{t} \tau^{k} e^{\mu \tau} d \tau .
\end{aligned}
$$

We then compute the following integral by integration by parts:

$$
\begin{aligned}
I_{k} & \equiv \int_{0}^{t} \tau^{k} e^{\mu \tau} d \tau=\frac{1}{\mu} t^{k} e^{\mu t}-\frac{k}{\mu} I_{k-1} \\
& =\frac{1}{\mu} t^{k} e^{\mu t}-\frac{k}{\mu^{2}} t^{k-1} e^{\mu t}+\frac{k(k-1)}{\mu^{2}} I_{k-2} \\
& =\frac{1}{\mu} t^{k} e^{\mu t}-\frac{k}{\mu^{2}} t^{k-1} e^{\mu t}+\cdots+\frac{(-1)^{k-1} k(k-1) \cdots 2}{\mu^{k-1}}\left(\frac{t}{\mu} e^{\mu t}-\frac{1}{\mu} I_{0}\right) \\
& =e^{\mu t} \sum_{l=1}^{k+1} \frac{(-1)^{l-1} k !}{\mu^{l}(k+1-l) !} t^{k+1-l}+\left(\frac{(-1)^{k+1} k !}{\mu^{k+1}}\right)
\end{aligned}
$$

with $I_{0}=\int_{0}^{t} e^{\mu \tau} d \tau=\frac{1}{\mu}\left(e^{\mu t}-1\right)$. 
Next, for (4), for $\lambda, \mu \in \mathbb{R}$ with $\lambda \neq \pm \mu$,

$$
\begin{aligned}
(\sin \lambda t * \cos \mu t)(t) & =\int_{0}^{t} \sin \lambda(t-\tau) \cos \mu \tau d \tau \\
& =\frac{1}{2} \int_{0}^{t}\{\sin (\lambda t-(\lambda-\mu) \tau)+\sin (\lambda t-(\lambda+\mu) \tau)\} d \tau \\
& =\frac{1}{2}\left[\frac{\cos (\lambda t-(\lambda-\mu) \tau)}{\lambda-\mu}+\frac{\cos (\lambda t-(\lambda+\mu) \tau)}{\lambda+\mu}\right]_{\tau=0}^{t} \\
& =\frac{\lambda}{\lambda^{2}-\mu^{2}} \cos \mu t-\frac{\lambda}{\lambda^{2}-\mu^{2}} \cos \lambda t \in A(\mathbb{R}) .
\end{aligned}
$$

If $\lambda= \pm \mu$, then $(\sin \lambda t * \cos \mu t)(t)=\frac{1}{2} t \sin \lambda t \in A(\mathbb{R})$.

Moreover, for $\lambda, \mu \in \mathbb{R}$ with $\lambda \neq \pm \mu$,

$$
\begin{aligned}
(\cos \lambda t * \cos \mu t)(t) & =\int_{0}^{t} \cos \lambda(t-\tau) \cos \mu \tau d \tau \\
& =\frac{1}{2} \int_{0}^{t}\{\cos (\lambda t-(\lambda-\mu) \tau)+\cos (\lambda t-(\lambda+\mu) \tau)\} d \tau \\
& =\frac{1}{2}\left[\frac{\sin (\lambda t-(\lambda-\mu) \tau)}{-(\lambda-\mu)}+\frac{\sin (\lambda t-(\lambda+\mu) \tau)}{-(\lambda+\mu)}\right]_{\tau=0}^{t} \\
& =\frac{\mu}{\lambda^{2}-\mu^{2}} \sin \mu t-\frac{\lambda}{\lambda^{2}-\mu^{2}} \sin \lambda t \in A(\mathbb{R}) .
\end{aligned}
$$

If $\lambda= \pm \mu$, then $(\cos \lambda t * \cos \mu t)(t)=\frac{1}{2} t \cos \lambda t+\frac{1}{2 \lambda} \sin \lambda t \in A(\mathbb{R})$.

Furthermore, for $\lambda, \mu \in \mathbb{R}$ with $\lambda \neq \pm \mu$,

$$
\begin{aligned}
(\sin \lambda t * \sin \mu t)(t) & =\int_{0}^{t} \sin \lambda(t-\tau) \sin \mu \tau d \tau \\
& =\frac{-1}{2} \int_{0}^{t}\{\cos (\lambda t-(\lambda-\mu) \tau)-\cos (\lambda t-(\lambda+\mu) \tau)\} d \tau \\
& =\frac{-1}{2}\left[\frac{\sin (\lambda t-(\lambda-\mu) \tau)}{-(\lambda-\mu)}-\frac{\sin (\lambda t-(\lambda+\mu) \tau)}{-(\lambda+\mu)}\right]_{\tau=0}^{t} \\
& =\frac{\lambda}{\lambda^{2}-\mu^{2}} \sin \mu t-\frac{\mu}{\lambda^{2}-\mu^{2}} \sin \lambda t \in A(\mathbb{R}) .
\end{aligned}
$$

If $\lambda= \pm \mu$, then $(\sin \lambda t * \sin \mu t)(t)=\frac{-1}{2} t \cos \lambda t+\frac{1}{2 \lambda} \sin \lambda t \in A(\mathbb{R})$.

Next, for (5), for $\mu, \lambda \in \mathbb{R}$ with $\mu \neq 0$,

$$
\left(e^{\mu t} * \sin \lambda t\right)(t)=e^{\mu t} \int_{0}^{t} e^{-\mu \tau} \sin \lambda \tau d \tau
$$


with the integral $I_{s} \equiv \int_{0}^{t} e^{-\mu \tau} \sin \lambda \tau d \tau$ computed as

$$
\begin{aligned}
I_{s} & =\left[\frac{e^{-\mu \tau}}{-\mu} \sin \lambda \tau\right]_{\tau=0}^{t}+\frac{\lambda}{\mu} \int_{0}^{t} e^{-\mu \tau} \cos \lambda \tau d \tau \\
& =\frac{e^{-\mu t}}{-\mu} \sin \lambda t+\frac{\lambda}{\mu^{2}}\left(1-e^{\mu t} \cos \lambda t\right)-\frac{\lambda^{2}}{\mu^{2}} I_{s}
\end{aligned}
$$

so that

$$
I_{s}=\frac{1}{\lambda^{2}+\mu^{2}}\left\{\lambda-e^{-\mu t}(\mu \sin \lambda t+\lambda \cos \lambda t)\right\}
$$

Similarly,

$$
\left(e^{\mu t} * \cos \lambda t\right)(t)=e^{\mu t} \int_{0}^{t} e^{-\mu \tau} \cos \lambda \tau d \tau
$$

with the integral $I_{c} \equiv \int_{0}^{t} e^{-\mu \tau} \cos \lambda \tau d \tau$ computed as

$$
\begin{aligned}
I_{c} & =\left[\frac{e^{-\mu \tau}}{-\mu} \cos \lambda \tau\right]_{\tau=0}^{t}-\frac{\lambda}{\mu} \int_{0}^{t} e^{-\mu \tau} \sin \lambda \tau d \tau \\
& =\frac{1}{\mu}\left(1-e^{-\mu t} \cos \lambda t\right)+\frac{\lambda}{\mu^{2}} e^{-\mu t} \sin \lambda t-\frac{\lambda^{2}}{\mu^{2}} I_{c}
\end{aligned}
$$

so that

$$
I_{c}=\frac{1}{\lambda^{2}+\mu^{2}}\left\{\mu-e^{-\mu t}(\mu \cos \lambda t-\lambda \sin \lambda t)\right\} .
$$

On the other hand, for (6), for $n \geq 0$ in $\mathbb{N}$ and $\lambda \in \mathbb{R}$ with $\lambda \neq 0$,

$$
\begin{aligned}
& \left(t^{n} * \sin \lambda t\right)(t)=\int_{0}^{t}(t-\tau)^{n} \sin \lambda \tau d \tau= \\
& \int_{0}^{t} \sum_{k=0}^{n}\left(\begin{array}{l}
n \\
k
\end{array}\right) t^{n-k}(-1)^{k} \tau^{k} \sin \lambda \tau d \tau=\sum_{k=0}^{n}\left(\begin{array}{l}
n \\
k
\end{array}\right) t^{n-k}(-1)^{k} \int_{0}^{t} \tau^{k} \sin \lambda \tau d \tau .
\end{aligned}
$$

We then compute the following integral by integration by parts:

$$
\begin{aligned}
I_{k} \equiv \int_{0}^{t} \tau^{k} \sin \lambda \tau d \tau & =\frac{-1}{\lambda} t^{k} \cos \lambda t+\frac{k}{\lambda} \int_{0}^{t} \tau^{k-1} \cos \lambda \tau d \tau \\
& =\frac{-1}{\lambda} t^{k} \cos \lambda t+\frac{k}{\lambda^{2}} t^{k-1} \sin \lambda t-\frac{k(k-1)}{\lambda^{2}} I_{k-2} .
\end{aligned}
$$

Inductively, if $k=2 m$ with $m \in \mathbb{N}$ and $m \geq 0$, then

$$
\begin{aligned}
& I_{2 m}=\sum_{l=1}^{m} \frac{(-1)^{l} k !}{\lambda^{2 l-1}(k-2 l+2) !} t^{k-2 l+2} \cos \lambda t \\
& +\sum_{l=1}^{m} \frac{(-1)^{(l-1)} k !}{\lambda^{2 l}(k-2 l+1) !} t^{k-2 l+1} \sin \lambda t+\frac{(-1)^{m} k !}{\lambda^{2 m}} I_{0}
\end{aligned}
$$


with $\mathrm{I}_{0}=\int_{0}^{\mathrm{t}} \sin \lambda \tau d \tau=\frac{1}{\lambda}-\frac{1}{\lambda} \cos \lambda t$, and hence,

$$
\begin{aligned}
I_{2 m}= & \sum_{l=1}^{m+1} \frac{(-1)^{l} k !}{\lambda^{2 l-1}(k-2 l+2) !} t^{k-2 l+2} \cos \lambda t \\
& +\sum_{l=1}^{m} \frac{(-1)^{(l-1)} k !}{\lambda^{2 l}(k-2 l+1) !} t^{k-2 l+1} \sin \lambda t+\frac{(-1)^{m} k !}{\lambda^{2 m+1}} .
\end{aligned}
$$

If $k=2 m+1$ with $m \in \mathbb{N}$ and $m \geq 0$, then

$$
\begin{aligned}
\mathrm{I}_{2 \mathrm{~m}+1}= & \sum_{l=1}^{\mathrm{m}} \frac{(-1)^{\mathrm{l}} \mathrm{k} !}{\lambda^{2 l-1}(k-2 l+2) !} \mathrm{t}^{\mathrm{k}-2 \mathrm{k}+2} \cos \lambda \mathrm{t} \\
& +\sum_{l=1}^{\mathrm{m}} \frac{(-1)^{(l-1)} k !}{\lambda^{2 l}(k-2 l+1) !} \mathrm{t}^{\mathrm{k}-2 l+1} \sin \lambda t+\frac{(-1)^{\mathrm{m}} \mathrm{k!}}{\lambda^{2 m}} \mathrm{I}_{1}
\end{aligned}
$$

with $I_{1}=\int_{0}^{t} \tau \sin \lambda \tau d \tau=\frac{-1}{\lambda} t \cos \lambda t+\frac{1}{\lambda^{2}} \sin \lambda t$, and hence,

$$
\begin{aligned}
I_{2 m+1}= & \sum_{l=1}^{m+1} \frac{(-1)^{l} k !}{\lambda^{2 l-1}(k-2 l+2) !} t^{k-2 l+2} \cos \lambda t \\
& +\sum_{l=1}^{m+1} \frac{(-1)^{(l-1)} k !}{\lambda^{2 l}(k-2 l+1) !} t^{k-2 l+1} \sin \lambda t .
\end{aligned}
$$

Similarly, for $n \geq 0$ in $\mathbb{N}$ and $\lambda \in \mathbb{R}$ with $\lambda \neq 0$,

$$
\begin{aligned}
& \left(t^{n} * \cos \lambda t\right)(t)=\int_{0}^{t}(t-\tau)^{n} \cos \lambda \tau d \tau= \\
& \int_{0}^{t} \sum_{k=0}^{n}\left(\begin{array}{l}
n \\
k
\end{array}\right) t^{n-k}(-1)^{k} \tau^{k} \cos \lambda \tau d \tau=\sum_{k=0}^{n}\left(\begin{array}{l}
n \\
k
\end{array}\right) t^{n-k}(-1)^{k} \int_{0}^{t} \tau^{k} \cos \lambda \tau d \tau .
\end{aligned}
$$

We then compute the following integral by integration by parts:

$$
\begin{aligned}
\mathrm{J}_{k} \equiv \int_{0}^{\mathrm{t}} \tau^{\mathrm{k}} \cos \lambda \tau d \tau & =\frac{1}{\lambda} \mathrm{t}^{\mathrm{k}} \sin \lambda t+\frac{-\mathrm{k}}{\lambda} \int_{0}^{\mathrm{t}} \tau^{\mathrm{k}-1} \sin \lambda \tau d \tau \\
& =\frac{1}{\lambda} \mathrm{t}^{\mathrm{k}} \sin \lambda t+\frac{\mathrm{k}}{\lambda^{2}} \mathrm{t}^{\mathrm{k}-1} \cos \lambda t-\frac{\mathrm{k}(\mathrm{k}-1)}{\lambda^{2}} \mathrm{~J}_{\mathrm{k}-2} .
\end{aligned}
$$

Inductively, if $k=2 m$ with $m \in \mathbb{N}$ and $m \geq 0$, then

$$
\begin{aligned}
J_{2 m}= & \sum_{l=1}^{m} \frac{(-1)^{(l-1)} k !}{\lambda^{2 l-1}(k-2 l+2) !} t^{k-2 l+2} \sin \lambda t \\
& +\sum_{l=1}^{m} \frac{(-1)^{(l-1)} k !}{\lambda^{2 l}(k-2 l+1) !} t^{k-2 l+1} \cos \lambda t+\frac{(-1)^{m} k !}{\lambda^{2 m}} J_{0}
\end{aligned}
$$


with $J_{0}=\int_{0}^{t} \cos \lambda \tau d \tau=\frac{1}{\lambda} \sin \lambda t$, and hence,

$$
\begin{aligned}
J_{2 m}= & \sum_{l=1}^{m+1} \frac{(-1)^{(l-1)} k !}{\lambda^{2 l-1}(k-2 l+2) !} t^{k-2 l+2} \sin \lambda t \\
& +\sum_{l=1}^{m} \frac{(-1)^{(l-1)} k !}{\lambda^{2 l}(k-2 l+1) !} t^{k-2 l+1} \cos \lambda t .
\end{aligned}
$$

If $k=2 m+1$ with $m \in \mathbb{N}$ and $m \geq 0$, then

$$
\begin{aligned}
J_{2 m+1}= & \sum_{l=1}^{m} \frac{(-1)^{(l-1)} k !}{\lambda^{2 l-1}(k-2 l+2) !} \mathrm{t}^{k-2 l+2} \sin \lambda t \\
& +\sum_{l=1}^{m} \frac{(-1)^{(l-1)} k !}{\lambda^{2 l}(k-2 l+1) !} \mathrm{t}^{k-2 l+1} \cos \lambda t+\frac{(-1)^{m} k !}{\lambda^{2 m}} J_{1}
\end{aligned}
$$

with $J_{1}=\int_{0}^{t} \tau \cos \lambda \tau d \tau=\frac{1}{\lambda} t \sin \lambda t+\frac{1}{\lambda^{2}}(\cos \lambda t-1)$, and hence,

$$
\begin{aligned}
J_{2 m+1}= & \sum_{l=1}^{m+1} \frac{(-1)^{(l-1)} k !}{\lambda^{2 l-1}(k-2 l+2) !} t^{k-2 l+2} \sin \lambda t \\
& +\sum_{l=1}^{m+1} \frac{(-1)^{(l-1)} k !}{\lambda^{2 l}(k-2 l+1) !} t^{k-2 l+1} \cos \lambda t+\frac{(-1)^{m+1} k !}{\lambda^{2 m+2}} .
\end{aligned}
$$

Finally, for (7), the convolution of general monomials is given by, as an example,

$$
\begin{aligned}
& \left(t^{n} e^{\mu_{1} t} \sin \lambda_{1} t\right) *\left(t^{m} e^{\mu_{2} t} \cos \lambda_{2} t\right)(t) \\
& =\int_{0}^{t}(t-\tau)^{n} e^{\mu_{1}(t-\tau)} \sin \lambda_{1}(t-\tau) \tau^{m} e^{\mu_{2} \tau} \cos \lambda_{2} \tau d \tau \\
& =e^{\mu_{1} t} \sum_{k=0}^{n}\left(\begin{array}{l}
n \\
k
\end{array}\right) t^{n-k}(-1)^{k} \int_{0}^{t} \tau^{k+m} e^{\left(\mu_{2}-\mu_{1}\right) \tau} \sin \lambda_{1}(t-\tau) \cos \lambda_{2} \tau d \tau
\end{aligned}
$$

with $\sin \lambda_{1}(t-\tau) \cos \lambda_{2} \tau=\frac{1}{2}\left\{\sin \left(\lambda_{1} t-\left(\lambda_{1}-\lambda_{2}\right) \tau\right)+\sin \left(\lambda_{1} t-\left(\lambda_{1}+\lambda_{2}\right) \tau\right)\right\}$, and then the following integral is computed inductively as

$$
\begin{aligned}
& I_{k+m, \sin } \equiv \int_{0}^{t} \tau^{k+m} e^{\left(\mu_{2}-\mu_{1}\right) \tau} \sin \left(\lambda_{1} t-\left(\lambda_{1} \pm \lambda_{2}\right) \tau\right) d \tau \\
& =\left[\tau^{k+m} \int e^{\left(\mu_{2}-\mu_{1}\right) \tau} \sin \left(\lambda_{1} t-\left(\lambda_{1} \pm \lambda_{2}\right) \tau\right) d \tau\right]_{\tau=0}^{t} \\
& \quad-(k+m) \int_{0}^{t} \tau^{k+m-1}\left(\int e^{\left(\mu_{2}-\mu_{1}\right) \tau} \sin \left(\lambda_{1} t-\left(\lambda_{1} \pm \lambda_{2}\right) \tau\right) d \tau\right) d \tau
\end{aligned}
$$


where the indefinite integral is computed by using integration by parts twice as:

$$
\begin{aligned}
& \int e^{\left(\mu_{2}-\mu_{1}\right) \tau} \sin \left(\lambda_{1} t-\left(\lambda_{1} \pm \lambda_{2}\right) \tau\right) d \tau \\
& =\frac{\mu_{2}-\mu_{1}}{\left(\mu_{2}-\mu_{1}\right)^{2}+\left(\lambda_{1} \pm \lambda_{2}\right)^{2}} e^{\left(\mu_{2}-\mu_{1}\right) \tau} \sin \left(\lambda_{1} t-\left(\lambda_{1} \pm \lambda_{2}\right) \tau\right) \\
& \quad+\frac{\lambda_{1} \pm \lambda_{2}}{\left(\mu_{2}-\mu_{1}\right)^{2}+\left(\lambda_{1} \pm \lambda_{2}\right)^{2}} e^{\left(\mu_{2}-\mu_{1}\right) \tau} \cos \left(\lambda_{1} t-\left(\lambda_{1} \pm \lambda_{2}\right) \tau\right)
\end{aligned}
$$

and hence, we obtain

$$
\begin{aligned}
& \mathrm{I}_{\mathrm{k}+\mathrm{m}, \sin }=\frac{\mu_{2}-\mu_{1}}{\left(\mu_{2}-\mu_{1}\right)^{2}+\left(\lambda_{1} \pm \lambda_{2}\right)^{2}} \mathrm{t}^{\mathrm{k}+\mathrm{m}} e^{\left(\mu_{2}-\mu_{1}\right) \mathrm{t}} \sin \left(\mp \lambda_{2} \mathrm{t}\right) \\
& +\frac{\lambda_{1} \pm \lambda_{2}}{\left(\mu_{2}-\mu_{1}\right)^{2}+\left(\lambda_{1} \pm \lambda_{2}\right)^{2}} \mathrm{t}^{\mathrm{k}+\mathrm{m}} e^{\left(\mu_{2}-\mu_{1}\right) \mathrm{t}} \cos \left(\mp \lambda_{2} \mathrm{t}\right) \\
& -\frac{(k+m)\left(\mu_{2}-\mu_{1}\right)}{\left(\mu_{2}-\mu_{1}\right)^{2}+\left(\lambda_{1} \pm \lambda_{2}\right)^{2}} \int_{0}^{t} \tau^{k+m-1} e^{\left(\mu_{2}-\mu_{1}\right) \tau} \sin \left(\lambda_{1} \mathrm{t}-\left(\lambda_{1} \pm \lambda_{2}\right) \tau\right) \mathrm{d} \tau \\
& -\frac{(k+m)\left(\lambda_{1} \pm \lambda_{2}\right)}{\left(\mu_{2}-\mu_{1}\right)^{2}+\left(\lambda_{1} \pm \lambda_{2}\right)^{2}} \int_{0}^{t} \tau^{k+m-1} e^{\left(\mu_{2}-\mu_{1}\right) \tau} \cos \left(\lambda_{1} t-\left(\lambda_{1} \pm \lambda_{2}\right) \tau\right) d \tau
\end{aligned}
$$

where the last two integrals are denoted by $I_{k+m-1, \sin }$ and $I_{k+m-1, \cos }$ respectively, and the integrals can be inductively reduced to the case of $\mathrm{I}_{0, \sin }$ and $\mathrm{I}_{0, \text { cos }}$ that are given by

$$
\begin{aligned}
& \mathrm{I}_{0, \sin } \equiv \int_{0}^{\mathrm{t}} e^{\left(\mu_{2}-\mu_{1}\right) \tau} \sin \left(\lambda_{1} \mathrm{t}-\left(\lambda_{1} \pm \lambda_{2}\right) \tau\right) d \tau \\
& =\frac{\mu_{2}-\mu_{1}}{\left(\mu_{2}-\mu_{1}\right)^{2}+\left(\lambda_{1} \pm \lambda_{2}\right)^{2}}\left\{e^{\left(\mu_{2}-\mu_{1}\right) \mathrm{t}} \sin \left(\mp \lambda_{2} \mathrm{t}\right)-\sin \left(\lambda_{1} \mathrm{t}\right)\right\} \\
& \quad+\frac{\lambda_{1} \pm \lambda_{2}}{\left(\mu_{2}-\mu_{1}\right)^{2}+\left(\lambda_{1} \pm \lambda_{2}\right)^{2}}\left\{e^{\left(\mu_{2}-\mu_{1}\right) \mathrm{t}} \cos \left(\mp \lambda_{2} \mathrm{t}\right)-\cos \left(\lambda_{1} \mathrm{t}\right)\right\}
\end{aligned}
$$

and

$$
\begin{aligned}
& \mathrm{I}_{0, \cos } \equiv \int_{0}^{\mathrm{t}} e^{\left(\mu_{2}-\mu_{1}\right) \tau} \cos \left(\lambda_{1} t-\left(\lambda_{1} \pm \lambda_{2}\right) \tau\right) d \tau \\
& =\frac{\mu_{2}-\mu_{1}}{\left(\mu_{2}-\mu_{1}\right)^{2}+\left(\lambda_{1} \pm \lambda_{2}\right)^{2}}\left\{e^{\left(\mu_{2}-\mu_{1}\right) t} \cos \left(\mp \lambda_{2} t\right)-\cos \left(\lambda_{1} t\right)\right\} \\
& \quad-\frac{\lambda_{1} \pm \lambda_{2}}{\left(\mu_{2}-\mu_{1}\right)^{2}+\left(\lambda_{1} \pm \lambda_{2}\right)^{2}}\left\{e^{\left(\mu_{2}-\mu_{1}\right) t} \sin \left(\mp \lambda_{2} t\right)-\sin \left(\lambda_{1} t\right)\right\} .
\end{aligned}
$$

Other cases of convolution products of general monomials with sin and cos changed are also computed similarly.

Theorem 5.3. The Laplace transform $\mathrm{L}$ is an algebra homomorphism from $\mathrm{A}(\mathbb{R})$ with convolution product to $\mathrm{R}_{0}(\mathbb{C})$ with point-wise multiplication.

Also, the inverse Laplace transform $\mathrm{L}^{-1}$ is an algebra homomorphism from $\mathrm{R}_{0}(\mathbb{C})$ to $A(\mathbb{R})$.

Then $\mathrm{L}^{-1} \circ \mathrm{L}=\operatorname{id}_{\mathrm{A}(\mathbb{R})}$ and $\mathrm{L} \circ \mathrm{L}^{-1}=\mathrm{id}_{\mathrm{R}_{\mathrm{O}}(\mathbb{C})}$ the identity maps on $\mathrm{A}(\mathbb{R})$ and $\mathrm{R}_{\mathrm{O}}(\mathbb{C})$. 
Remark. Note that we identify a rational function $g$ in $R_{0}(\mathbb{C})$ with the image of $f \in A(\mathbb{R})$ under $L$ with domain of convergence such that $\mathrm{L}(\mathrm{f})=\mathrm{g}$. Indeed, the infimum of the real part of the domain of convergence for $L(f)=g$ is defined to be the maximum of the real parts of poles of $g$, so that the domain of convergence for $L(f)$ is determined by $g$ uniquely.

Proof. It is well known that $\mathrm{L}(f * g)(s)=\mathrm{L}(\mathrm{f})(\mathrm{s}) \cdot \mathrm{L}(\mathrm{g})(\mathrm{s})$ for $f, g \in A(\mathbb{R})$. It follows by the convolution products checked explicitly in Proposition 5.2, in particular, that any element of $A(\mathbb{R})$, which is a linear combination of multiples of elementary continuous functions, is a continuous function on $\mathbb{R}$, so that, as also a well known fact, the Laplace transform is injective on $A(\mathbb{R})$ but restricted to $[0, \infty)$, and hence the inverse Laplace transform $L^{-1}$ is also injective on $L(A(\mathbb{R}))$. Note that real coefficients in such linear combinations are determined uniquely by the injectivity, so that we may extend the definition domains from $[0, \infty)$ to $\mathbb{R}$ preserving the injectivity. It is clear that $L(A(\mathbb{R}))$ is contained in $R_{0}(\mathbb{C})$ by using basic formulae in Laplace transform and by Proposition 5.2. For instance, check that

$$
\begin{aligned}
& \mathrm{L}\left(e^{\mu t} t^{n} \sin \lambda t\right)(s)=L\left(t^{n} \sin \lambda t\right)(s-\mu)= \\
& (-1)^{n} L\left((-t)^{n} \sin \lambda t\right)(s-\mu)=(-1)^{n} \frac{d^{n}}{d s^{n}}\left(\frac{\lambda}{(s-\mu)^{2}+\lambda^{2}}\right) \in R_{0}(\mathbb{C}) .
\end{aligned}
$$

The last belonging is proved by induction. Indeed, if $\frac{p(s)}{q(s)} \in R_{0}(\mathbb{C})$, then $\frac{d}{d s}\left(\frac{p(s)}{q(s)}\right)=\frac{p^{\prime}(s) q(s)-p(s) q^{\prime}(s)}{q(s)^{2}} \in$ $\mathrm{R}_{0}(\mathbb{C})$.

It is also checked explicitly in Theorem 4.1 that any element of $R_{0}(\mathbb{C})$ is mapped to an element of $A(\mathbb{R})$ under $L^{-1}$.

Corollary 5.4. It follows that the algebra $\mathrm{A}(\mathbb{R})$ with convolution product is isomorphic to $\mathrm{R}_{0}(\mathbb{C})$, as an algebra.

It also follows that the algebra $\mathrm{A}(\mathbb{R})$ with point-wise multiplication is isomorphic to $\mathrm{R}_{0}(\mathbb{C})$, as a real vector space.

Remark. The Laplace transform L (as well as the inverse $\mathrm{L}^{-1}$ ) is linear but dose not preserve point-wise multiplication. For instance,

$$
\mathrm{L}\left(\mathrm{t}^{2}\right)=\frac{2}{\mathrm{~s}^{3}} \neq \mathrm{L}(\mathrm{t}) \cdot \mathrm{L}(\mathrm{t})=\frac{1}{\mathrm{~s}^{2}} \cdot \frac{1}{\mathrm{~s}^{2}}=\frac{1}{\mathrm{~s}^{4}} .
$$

Received: October 2013. Accepted: August 2014.

\section{References}

[1] Hiroshi Fukawa, Laplace transformation and ordinary differential equations (in Japanese), Sho-Ko-dou (1995). 
[2] M. S. J., Mathematics Dictionary, (Sugaku Jiten, in Japanese), Math. Soc. Japan, 4th edition, Iwanami (2007).

[3] A. Vretblad, Fourier Analysis and Its Applications, GTM 223, Springer (2003). 